\title{
FUNGOS EMBOLORADORES E MANCHADORES DE MADEIRA EM TORAS ESTOCADAS EM INDÚSTRIAS MADEIREIRAS NO MUNICÍPIO DE MANAUS, AMAZONAS, BRASIL
}

\author{
Rogério Eiji HANADA ${ }^{1}$, Ceci SALES-CAMPOS ${ }^{1}$, Raimunda Liége Souza de \\ ABREU' ${ }^{1}$, Ludwig PFENNING
}

\begin{abstract}
RESUMO - Visando conhecer a diversidade e a incidência de fungos emboloradores e manchadores de madeira da região Amazônica, foi realizado um levantamento em 12 espécies florestais, estocadas em quatro indústrias madeireiras de Manaus. De cada espécie florestal selecionaram-se cinco toras, das quais retiraram-se amostras de onde procedeu-se isolamento dos fungos. Foram isolados 106 fungos associados às essências florestais, representados por nove gêneros e por dezesseis espécies. Paecilomyces variotii e Lasiodiplodia theobromae foram as espécies fúngicas mais freqüentemente associadas às espécies florestais. Ceiba pentandra e Hura crepitans foram as essências florestais que apresentaram maior diversidade de fungos, com sete espécies cada uma. Hymeneae courbaril foi a que apresentou menor diversidade de fungo, com apenas uma espécie.
\end{abstract}

Palavras-chave: madeiras da Amazônia, diversidade fúngica, preservação da madeira

\section{Mould and stain fungi in logs stored in wood industries of Manaus, Amazonas, Brazil}

\begin{abstract}
In order to know a diversity and incidence of wood mould and wood stain fungi from Amazonian wood, the survey and identification of these kinds of fungi were carried out in twelve wood species from four wood industries of Manaus, Amazonas. Five logs were randomly selected. Wood samples were prepared and transported to the Wood Pathology Laboratory at the Forest Product Research Center of the National Institute for Amazonian Research, where the isolation and identification of the fungi were conducted. One hundred and six fungi associated to the wood species were isolated, represented by nine genera and sixteen fungi species. Paecilomyces variotti and Lasiodiplodia theobromae were the more representative fungi, associated with ten and seven wood species respectively. Ceiba pentandra e Hura crepitans were the wood species with highest diversity of fungi, with seven species each. Hymenae courbaril was the wood that presented the lowest diversity of fungi, with only one fungus.
\end{abstract}

Key-words: Amazonian wood industries, diversity of fungi, wood preservation

\section{INTRODUÇÃO}

A madeira representa o principal produto florestal e é, sem dúvida, um dos materiais orgânicos mais importantes e complexos que se conhece. Devido a sua complexidade anatômica e química pode sustentar uma rica comunidade de espécies de fungos e de outros microrganismos (Dix \& Webster, 1995).
Geralmente, os primeiros fungos que colonizam as árvores recém-abatidas são os emboloradores e os manchadores de madeira, devido a grande quantidade de substâncias de reserva, das quais eles se nutrem, e à elevada umidade. Dependendo da espécie florestal, dos fatores ambientais e dos tratamentos químico ou físico, os fungos podem ocupar toda a superfície da tora, em menos de 48 horas (Oliveira et al., 1986).

${ }^{1}$ Coordenação de Pesquisas em Produtos Florestais / Instituto Nacional de Pesquisas da Amazônia-CPPF/ INPA, Av. André Araújo, 2936, Caixa Postal 478, CEP 69.011-970, Manaus, AM, Brasil. E-mail: rhanada@inpa.gov.br. 2Departamento de Fitopatologia, Universidade Federal de Lavras, Caixa Postal 37, CEP 37.200-000, Lavras, MG, Brasil. 
A madeira atacada por fungos emboloradores apresenta, em sua superfície, área de aspecto pulverulento, de coloração variada, constituída de massa de esporos, facilmente removível por raspagem. Por outro lado, as hifas dos fungos podem penetrar profundamente no alburno sem afetar a coloração da madeira, pois as mesmas são hialinas (Oliveira et al., 1986). A madeira intensamente embolorada apresenta redução na sua resistência ao impacto, sendo que as demais propriedades mecânicas são pouco afetadas (Scheffer, 1973).

Os fungos manchadores, ao contrário, apresentam hifas pigmentadas ou hifas hialinas que secretam substâncias coloridas. A madeira atacada por estes fungos apresenta, no alburno, áreas de coloração variável, geralmente de azul a cinza escuro, que são observadas em cortes transversais e distribuem-se radialmente. As manchas, que podem ser superficiais ou profundas, depreciam a qualidade e o valor comercial da madeira (Oliveira et al., 1986). Alburno intensamente manchado pode apresentar reduções de $1 \%$ a $2 \%$ na densidade, de $2 \%$ a $10 \%$ na dureza, de $1 \%$ a $5 \%$ na resistência à flexão e de $15 \%$ a $30 \%$ ao impacto (Scheffer, 1973).

Sob condições favoráveis, todas as espécies florestais são suscetíveis ao ataque de fungos xilófagos. A distância do local de abate das árvores ao local de beneficiamento da madeira, a temperatura e a umidade elevadas da região Amazônica, favorecem a incidência de microrganismos (Sales-Campos et al., 2000). Por outro lado, os tratamentos de preservação empregados pelas indústrias madeireiras no Amazonas são, na maioria das vezes, ineficientes devido a falta de informações sobre microrganismos que colonizam a madeira e o uso dos preservativos.

Diante deste contexto, realizou-se um levantamento, visando conhecer a diversidade e a incidência de fungos emboloradores e manchadores em espécies florestais utilizadas por indústrias madeireiras de Manaus.

\section{MATERIAL E MÉTODOS}

O levantamento foi conduzido em quatro indústrias madeireiras da cidade de Manaus, onde selecionaram-se cinco toras de árvores diferentes, de cada espécie, com sintomas de ataque de fungos emboloradores e manchadores. Foram selecionadas toras das seguintes espécies: Calophyllum brasiliense Cambess. (jacareúba), Ceiba pentandra (L.) Gaertn (sumaúma), Copaifera multijuga Hayne (copaíba), Couroupita guianensis Aubl. (macacarecuia), Erisma calcaratum (Link) Warm. (caferana), Hevea brasiliensis Müll. Arg. (seringueira), Hura creptans L. (assacu), Hymenaea courbaril (jatobá), Hymenolobium pulcherrimum Ducke (angelim pedra), Pseudobombax munguba (Mart \& Zucc.) Dugand. (munguba), Schefflera morototoni (Aubl.) Decne \& Planch. (morototó) e Virola surinamensis (Rol.) Warb. (virola). Do alburno de cada uma das toras, com auxílio de um formão, retiraram-se amostras de aproximadamente $5 \mathrm{~cm} \times 5 \mathrm{~cm} \times 2 \mathrm{~cm}$, as quais foram transferidas para sacos de papel.

No Laboratório de Patologia da Madeira do INPA, em Manaus, procedeu-se ao isolamento e à identificação dos fungos. Das referidas amostras retiraram-se três fragmentos de 10 a $20 \mathrm{~mm}^{2}$, os quais foram imersos em água destilada estéril por dois minutos e colocados sobre um papel de filtro estéril para retirar o excesso de água. Em seguida, os fragmentos foram semeados, de forma eqüidistante, em placas de Petri contendo meio de cultura malte-ágar à $3 \%$ e $250 \mathrm{ppm}$ de antibiótico cloranfenicol. As placas foram mantidas em incubadora, no escuro, a $25{ }^{\circ} \mathrm{C}+$ $2{ }^{\circ} \mathrm{C}$, por um período de aproximadamente 30 dias. Â medida que as colônias se desenvolviam, eram transferidas para tubos de ensaio contendo o mesmo meio de cultura. Estes, foram mantidos nas mesmas condições até o micélio ocupar toda, ou a maior parte da superfície do meio. Em seguida, as culturas foram armazenadas em geladeira a $5{ }^{0} \mathrm{C}$, para sua posterior identificação.

Para a identificação dos fungos foram feitas observações macroscópicas das culturas e microscópicas em lâminas semipermanentes preparada com lactofenol e corante azul de algodão. Sob microscópico óptico foram feitas medições das estruturas reprodutivas dos 
fungos. Para alguns fungos foram preparadas microculturas conforme descrito por Fernandez (1993), visando observar detalhes das estruturas, particularmente importantes na delimitação das espécies. As características macroscópicas e microscópicas foram comparadas com às descritas em bibliografia especializada (Rifai, 1969; Booth, 1971; Samson, 1974; Carmichael, et al. 1980; Klich \& Pitt, 1988; Pitt, 1991; Halin, 1997; 1998; Barnett \& Hunter, 1998).

\section{RESULTADOS E DISCUSSÃO}

Foram obtidos 106 isolados de fungos associados às amostras de madeira. Paecilomyces variotii Bainier foi o fungo de maior prevalência, isolado de dez espécies florestais analisadas, seguido de Lasiodiplodia theobromae (Pat.) Griff. \& Maubl., isolado de sete espécies. O gênero Trichoderma foi o que apresentou maior diversidade de espécies, tendo sido identificadas quatro (Tabela 1). Todos os fungos obtidos neste levantamento pertencem ao grupo Ascomicotina ou são fungos Mitospóricos. Geralmente, fungos desses grupos são responsáveis por bolores e manchas encontrados em madeira (Oliveira et al., 1986).

As espécies com madeira de baixa densidade como C. pentandra e H. creptans, S. morototoni, P. munguba e $V$. surinamensis (Loureiro et al., 1997; Vasconcelos et al., 2001) foram as que apresentaram maior diversidade $\mathrm{e}$

Tabela 1. Incidência de fungos emboloradores e manchadores em toras de madeiras estocadas em indústrias madeireiras de Manaus.

\begin{tabular}{|c|c|c|c|c|c|c|c|c|c|c|c|c|}
\hline \multirow[t]{2}{*}{ Fungos } & \multicolumn{12}{|c|}{ Incidência (\%) } \\
\hline & $\mathrm{Cb}^{*}$ & $\mathrm{Cp}$ & $\mathrm{Cm}$ & $\mathrm{Cg}$ & Ec & $\mathrm{Hb}$ & $\mathrm{Hc}$ & $\mathrm{Hc}_{1}$ & $\mathrm{Hp}$ & $\mathrm{Pm}$ & $\mathrm{Sm}$ & Vs \\
\hline Aspergillus flavus Link & 0 & 0 & 0 & 0 & 0 & 0 & 0 & 0 & 60 & 0 & 40 & 40 \\
\hline Aspergillus niger Van Tieghen & 0 & 0 & 0 & 0 & 0 & 0 & 60 & 0 & 0 & 60 & 40 & 0 \\
\hline Fusarium moniliforme Sheldon & 0 & 0 & 0 & 0 & 0 & 0 & 20 & 0 & 0 & 0 & 0 & 0 \\
\hline Fusarium oxysporum Schelecht & 0 & 20 & 0 & 0 & 0 & 0 & 0 & 0 & 0 & 0 & 0 & 0 \\
\hline $\begin{array}{l}\text { Lasiodiplodia theobromae (Pat.) Griff. } \\
\text { el Maubl. }\end{array}$ & 0 & 60 & 0 & 40 & 40 & 60 & 60 & 0 & 0 & 0 & 60 & 60 \\
\hline Nectria sp. & 20 & 0 & 0 & 0 & 0 & 0 & 0 & 0 & 0 & 0 & 0 & 0 \\
\hline Paecilomyces variotii Bainier & 0 & 100 & 20 & 40 & 60 & 60 & 80 & 20 & 0 & 80 & 80 & 80 \\
\hline Penicillium citrinum Thom & 0 & 20 & 40 & 0 & 0 & 0 & 20 & 0 & 0 & 0 & 0 & 40 \\
\hline Penicillium chysogenum Thom & 0 & 0 & 0 & 0 & 0 & 0 & 0 & 0 & 40 & 40 & 0 & 0 \\
\hline Penicilium janthinellun Biourge & 0 & 0 & 0 & 40 & 0 & 0 & 40 & 0 & 0 & 0 & 0 & 0 \\
\hline Sordaria sp. & 0 & 40 & 0 & 0 & 0 & 0 & 0 & 0 & 0 & 40 & 0 & 0 \\
\hline Trichoderma harzianum Rifai & 0 & 0 & 0 & 0 & 0 & 20 & 0 & 0 & 0 & 60 & 40 & 40 \\
\hline Trichoderma longibrachiatum Rifai & 40 & 0 & 0 & 0 & 0 & 0 & 0 & 0 & 0 & 0 & 0 & 0 \\
\hline Trichoderma pseudokonigii Rifai & 0 & 0 & 0 & 0 & 40 & 0 & 0 & 0 & 0 & 0 & 20 & 0 \\
\hline Trichoderma viride Pers. ex S.F. Gray & 0 & 40 & 0 & 0 & 0 & 0 & 60 & 0 & 0 & 0 & 0 & 0 \\
\hline Westerdijkella sp. & 0 & 40 & 0 & 0 & 0 & 0 & 0 & 0 & 0 & 0 & 0 & 0 \\
\hline
\end{tabular}

*(Cb) Calophyllum brasiliensis, $(\mathrm{Cp})$ Ceiba pentandra, $(\mathrm{Cm})$ Copaifera multijuga, $(\mathrm{Cg})$ Couroupita guianensis, (Ec) Erisma calcaratum, (Hb) Hevea brasiliensis, (Hc) Hura creptans, $\left(\mathrm{Hc}_{1}\right)$ Hymenea courbaril, (Hp) Hymenolobium pulcherrimum, (Pm) Pseudobombax munguba, (Sm) Schefflera morototoni, (Vs) Virola surinamensis.

incidência de fungos (Figura 1). Segundo Panshin \& De Zeeuw (1980), a variabilidade existente nas propriedades físicas e mecânicas, pode estar relacionada com a composição e a quantidade de extrativos existentes na madeira. Esses extrativos vão conferir maior ou menor resistência a fungos degradadores. No caso de fungos emboloradores e manchadores, a presença de lignina, em alta quantidade, torna-se um fator limitante para o estabelecimento desses organismos, uma vez que esses fungos geralmente não conseguem degradar a lignina (Lepage, 1986). 


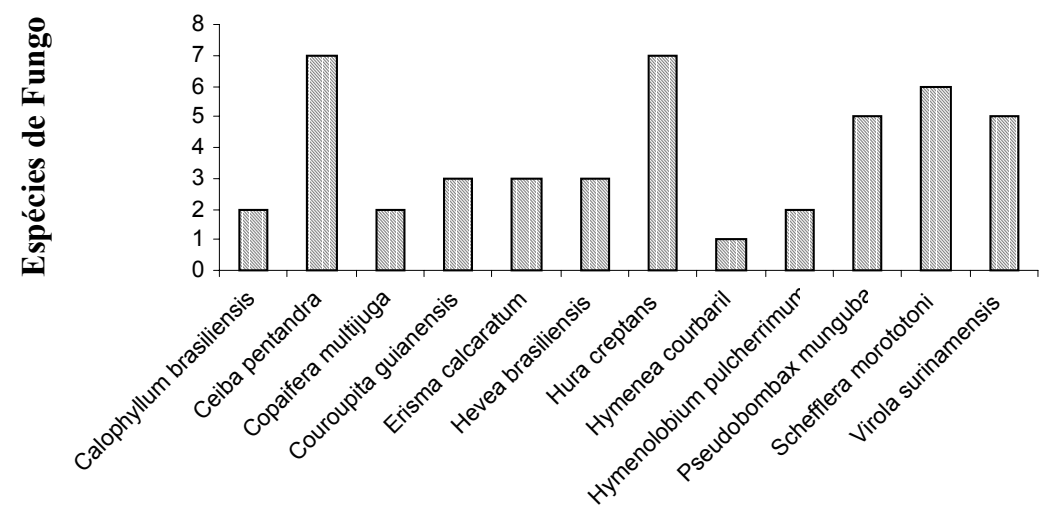

Espécies Florestais

Figura 1. Número de espécies de fungos manchadores ou emboloradores associados com toras de madeiras armazenadas em serrarias de Manaus, AM.

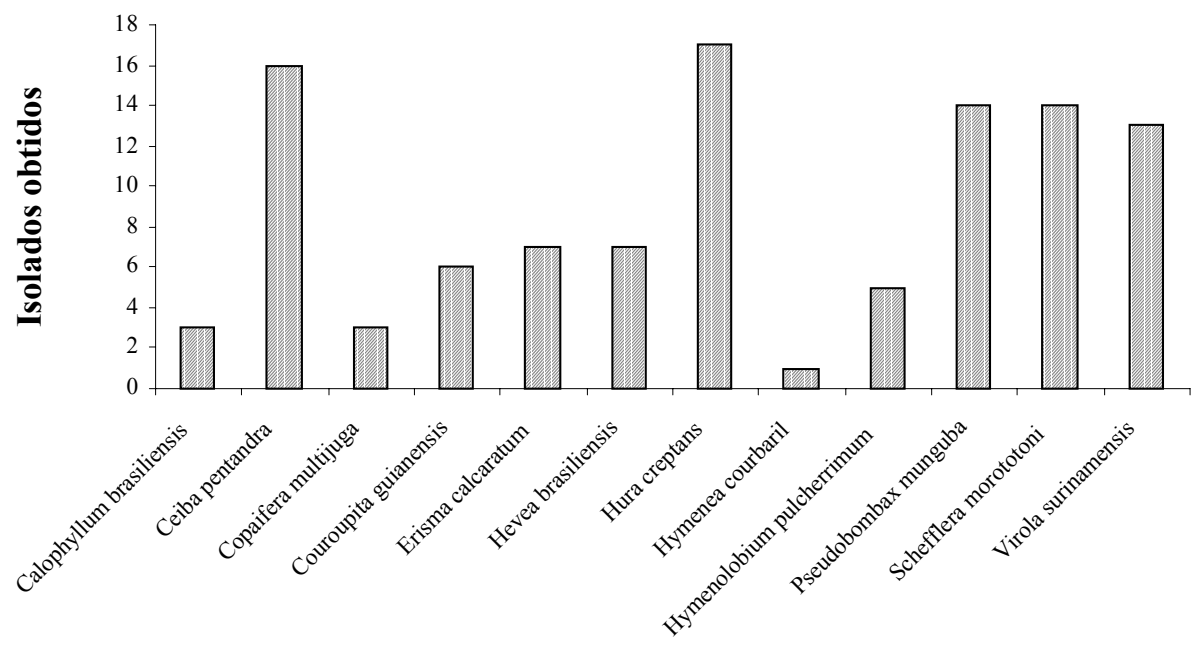

\section{Espécies Florestais}

Figura 2. Número de isolados de fungos emboloradores e manchadores de madeira obtidos de toras de diferentes espécies armazenadas, em Manaus, AM.

Muitas das espécies de fungos emboloradores e ou manchadores de madeira encontradas no levantamento já haviam sido relatadas ocorrendo em outras espécies florestais. A espécie $P$. variotii, é citada por Bauch et al. (1991) como agente causal da descoloração ou mancha amarela de Quercus sp. $\mathrm{Na}$ África e no Brasil L. theobromae foi relatado como agente causal da mancha azul das madeiras (Fourgerousse, 1958, Encinas, 1996).
Dix \& Webster (1995) citaram os fungos Trichoderma spp., Fusarium sp., Paecylomyces sp. e Penicillium spp. como emboloradores de madeira. Ye et al. (1993) isolaram os fungos Trichoderma, Fusarium, Aspergillus, Penicillium, Sordaria, entre outros, de Pinus e Elwy (1999) isolou Aspergillus flavus, a partir de madeira em decomposição. Hanlin (1997) cita que muitas espécies de Nectria são encontradas em várias espécies florestais e, que espécies de 
Westerdykella são saprofitas e geralmente ocorrem no solo. Provavelmente este é o primeiro relato de Westerdykella associado à madeira.

Não foi possível classificar os fungos isolados neste trabalho como emboloradores ou manchadores. Dez espécies, A. niger, $F$. moniliforme, $F$. oxysporum, $L$. theobromae, $P$. variotii, $P$. chysogenum, T. harzianum, $T$. longibrachiatum, T. pseudokonigii e T. viride, foram isoladas a partir de amostras apresentando coloração cinza ou azul. No entanto, isso não significa que os demais fungos isolados são emboloradores de madeira. Conforme Käärik (1975), uma mesma espécie de fungo pode atuar de forma diferente em diferentes circunstâncias. Além disso, os fungos emboloradores e manchadores ocorrem quase que concomitantemente, ocupando nichos ecológicos bastante próximos (Oliveira et al., 1986). Käärik (1975) acrescenta ainda que, geralmente, a distinção entre fungos emboloradores e manchadores está baseada em suas atividades enzimáticas, as quais diferenciam os principais grupos fisiológicos que preenchem sucessivamente os diferentes nichos ecológicos existentes na madeira, não discriminando, necessariamente, grupamentos taxonômicos. Portando, nem sempre é possível separar ou discernir com clareza se o fungo provoca bolor ou mancha na madeira, sem um estudo histológico.

Sob certas circunstâncias, fungos emboloradores e manchadores podem ser antagônicos a fungos degradadores, principalmente se eles forem os colonizadores pioneiros (Hulme \& Shields, 1975). Nesta última década, vários estudos explorando essa linha de pesquisa têm sido realizados. Brown \& Bruce (1998), estudaram o potencial do Trichoderma viride como antagonista a fungos degradadores de madeira. Schoeman et al. (1993) observaram que T. harzianum reduziu a quantidade de fungos apodrecedores em toras de Pinus sp. e Messner et al. (1996) observaram que madeiras infestadas com $T$. harzianum mostraram resistência a fungos degradadores, principalmente aos fungos da podridão parda.

Embora o escopo do trabalho não tenha sido avaliar os danos ocasionados pelos fungos em madeira, é importante ressaltar que a maioria dos fungos isolados, causam manchas que comprometem a qualidade e, conseqüentemente, o valor comercial. Portando, é torna-se fundamental um estudo da avaliação dos danos provocados pelos fungos xilófagos e as perdas econômicas decorrentes do ataque desses organismos em toras estocadas nas indústrias madeireiras.

\section{CONCLUSÕES}

A grande quantidade e diversidade da micoflora isolada neste levantamento indicam que as indústrias madeireiras da região de Manaus não estão estocando adequadamente as madeiras. Foi isolada pelo menos uma espécie de fungo manchador ou embolorador de cada amostra de madeira estudada, sendo que as madeiras de baixa densidade foram as mais suscetíveis ao ataque desses fungos. Os fungos $P$. variotii e $L$. teobromae foram os menos específicos, sendo o primeiro, o de maior prevalência.

\section{BIBLIOGRAFIA CITADA}

Barnett, H.L.; Hunter, B.B. 1998. Illustrated genera of imperfect fungi. $4^{\text {th }}$ ed. The American Phytopathological Society, St. Paul, Minnesota. 218p.

Bauch, J.; Hundt, H.; Weissmann, G.; Lange, W.; Jubel, H.; Von-Hundt, H. 1991. On the causes of yellow discoloration of oak heartwood (Quercus sect. Robur) during drying. Holzforschung, 45(2): 79-85.

Booth, C. 1971. The Genus Fusarium. Common. Mycological Institute. Kew, England. $237 \mathrm{p}$.

Brown, H.L.; Bruce, A. 1998. Assessments of the biocontrol potential of a Trichoderma viride isolate in a field trial. In $20^{\text {th }} \mathrm{An}$ nual Meeting of the IRG, Maastricht, Netherlands, June. $18 \mathrm{p}$.

Carmichael, J.W, BryceKedrick, W., Conners, I.L.; Sigler, L. 1980. General of Hyphomycetes. The University of Alberta Press, Canada. 386p.

Dix, N.J.; Webster, J. 1995. Fungal Ecology. Chapman \& Hall, London. 549p.

Elwy, E. 1999. Growth and cellulolytic activity of certain aspergilli isolated from ancient wood. African J. Mycol. Biotech., 7(1): 47-55.

Encinas, O. 1996. Development and significance of attack by Lasiodiplodia 
theobromae (Pat.) Griff. \& Maubl. in Caribbean pine wood and some other wood species. Acta Universitatis, 8:107.

Fernandez, M.R. 1993. Manual para laboratório de fitopatologia. EmbrapaCNPT, Passo Fundo. 128p.

Fourgerousse, M. 1958. Les alteration fungiques des bois frais em Afrique Tropicale et plus particulierment de l'llomba et du Limba. Bois et Forest de Tropiques, 60: 41-56.

Hanlin, R.T. 1997. Illustrated Genera Ascomycetes. Vol. 1. The American Phytopathological Society, USA. 263p.

Hanlin, R.T. 1998. Illustrated Genera Ascomycetes. Vol. 2. The American Phytopathological Society, USA. 258p.

Hulme, M.A.; Shields, J.K. 1975. Antagonistic and synergistic effects for biological control of decay, In: Liese, W. (ed.) Biological Transformation of Wood by Microorganism. Springer-Verlag, Berlin, p.52-63.

Käärik, A. 1975. Decomposition of wood. In: Dickinson, C.H.; Pugh, G.J.F. (eds.). Biology of plant litter decomposition. Vol. 1. Academic Press, London. p.129-174.

Klich, M.A.; Pitt, J.I. 1988. A Laboratory guide to the common Aspergillus species and their teleomorphs. Commonwealth Scientific an Industrial Research Organization, Australia. 116p.

Lepage, E.S. 1986. Química da madeira. In: Lepage. E.S. (ed.) Manual de Preservação de Madeiras. Vol. 1. IPT, São Paulo. p. 69-97.

Loureiro, A. A.; Freitas, J.A.; Freitas, C.A A. 1997. Essências Madeireiras da Amazônia 3. MCT/INPA/CPPF. 205p.

Messner, K.; Burgel, J.; Horvath, E.M.; Schlick, A.; Fleck, V.; Marchler, A. 1996. State of development of the LCT method of wood preservation. Holz. Zentralblatt, 122(15): 232-233.
Oliveira, A.M.F., Lelis, A.T de, Lepage, E.S., Lopes, G.A.C., Oliveira, L.C.S., Cañedo, M.D., Milano, S. 1986. Agentes destruidores de madeira. In: Lepage, E.S. (ed.) Manual de Preservação de madeiras. Vol. 1. IPT, São Paulo. p.99-278.

Panshin, A.J.; De Zeewun, C. 1980. Textbook of technology. $3^{\text {th }}$ ed. McGraw Hill, New York. 722p.

Pitt. J.I. 1991. A Laboratory guide to common Penicillium species. Commonwealth Scientific and Industrial, Research Organization, Division of Food Processing, 187p.

Rifai, M.A. 1969. A revision of the Genus Trichoderma. Mycological Papers, Indonésia, 116: 1-56.

Sales-Campos, C.; Abreu, R.L. S.; Vianez, B.F. 2000. Condições de uso e processamento de madeiras nas indústrias madeireiras de Manaus, Amazonas, Brasil. Acta Amazonica, 30(2): 319-331.

Samson, R. 1974. Paecilomyces and some allied Hyphomycetes. Studies in Mycology, 6: 1-119.

Scheffer, T.C. 1973. Microbiological degradation and the causal organism. In: Nicholas, D.D. (ed.) Wood deterioration and its prevention by preservative treatments. Vol.1. Syracuse University Press, Syracuse. p. 31-106.

Schoeman, M.W.; Webber, J.F.; Dickinson, D.J. 1993. Chain-saw application of Trichoderma harzianum Hifai to reduce fungal deterioration of freshly felled pine logs. Mat. Org., 28(4): 243-250.

Vasconcelos, F.J; Freitas, J.A; Lima, V.M.O.C; Moteiro, L.V; Pereira, S.J. 2001. Madeiras tropicais de uso industrial do Maranhão: Características tecnológicas. INPA/ UFMA. 96p.

Ye, W.; Zhang, Q.; Hong, S.; Zhu, D. 1993. Studies on fungi associated with Bursaphelenchus xylophilus on Pinus massoniana in Shenzhen, China. Afro Asian J. Nem., 3(1): 47-49.

\section{Recebido: 19/02/2002}

